Ann. Génét. Sél. anim., I980, 12 (4), 32I-342.

\title{
Les variants colorés en race Mérinos d'Arles I. Étude de la génération parentale
}

\author{
J. J. LAUVERGNE (*), Melinda \\ J. BURRILL (**) et A. SADORGE (***) \\ (*) Laboratoire de Génétique Factoriblle, I.N.R.A., \\ $7835^{\circ}$ Jouy-en-Josas, France, \\ Centre national de Recherches zootechniques \\ (**) Department of Animal Science, California State Polytechnic, \\ University, Pomona, California 9I768, U.S.A. \\ (***) Éleveur, ro, rue Théophile-Rives, 13200 Arles, France
}

\section{Résumé}

Les enquêtes faites en I979 sur l'agnelage d'automne 78 et en I 980 sur celui d'automne 79 ont porté sur 8 troupeaux inscrits et sur 27 troupeaux non inscrits de Mérinos d'Arles qui hivernent en Crau et en Camargue et estivent dans les Alpes. Il s'agissait de préciser la situation démographique de la race en vue d'une étude de génétique mendélienne et de génétique des populations des variants colorés.

La taille moyenne des troupeaux était en 78 et 79 de 786 et 800 brebis pour les troupeaux inscrits et de 513 et 560 brebis pour les troupeaux non inscrits. Les effectifs des mâles de service correspondants étaient respectivement de 29,28 , I 6 et 16.

La distribution de l'effectif des troupeaux non inscrits est log normale, aussi bien pour les mâles que pour les femelles.

On compte une moyenne de 27 à 28,5 brebis par bélier à la lutte pour les troupeaux inscrits, de 33 à 35,4 brebis par bélier pour les troupeaux non inscrits. La liaison de cette variable avec la taille des troupeaux n'est pas régulière et semble révéler une certaine diversité dans les conduites d'élevage. La prolificité des brebis à l'agnelage d'automne variait entre $\mathrm{I}, 04$ et $\mathrm{I}, \mathbf{1} 8$.

L'échange de matériel génétique pour le sexe mâle est limité : I 2 p. roo, aussi bien pour les troupeaux inscrits que non inscrits, bien que les troupeaux soient assez proches géographiquement pendant l'hivernage au cours duquel se fait la lutte de printemps. Des transferts non contrôlés de gènes extérieurs ont lieu en outre à la faveur de l'estive où les troupeaux Mérinos sont mélangés à d'autres troupeaux, mais leur importance semble limitée.

Dans les troupeaux inscrits, seuls les reproducteurs blancs sont admis, à trois exceptions près (des brebis). Dans les troupeaux non inscrits, où tous les mâles reproducteurs sont blancs, on observe des brebis rouges $(0,03$ et $0,02 \mathrm{p}$. Ioo resp. en 1978 et 79$)$, noires ou pie noir $(0,4$ et 0,8 p. roo resp.), ou blaireau (o,0o8 et 0,3 p. roo resp.), le total des brebis colorées était de 0,4 en 1978 et de r,2 p. Ioo en I979. Il y a en outre quelques floucats (mâles castrés) de couleur : 0,3 p. 100 en 1979, lors de l'enquête 1980.

La motivation de la conservation d'adultes colorés ne semble pas être le comptage des brebis en pâture alors que l'existence de floucats (colorés ou non) semble correspondre assez nettement, parmi les troupeaux inscrits tout au moins, à une utilisation comme conducteur du troupeau. Dans ces conditions, on pense que cette conservation d'animaux colorés est une survivance de pratiques ancestrales. 


\section{I. - Introduction}

Dans toutes les races de moutons blancs il naît de temps en temps des animaux colorés et plusieurs questions se posent. S'agit-il de mutations récurrentes ou de gènes maintenus grâce à un avantage sélectif des hétérozygotes? Doit-on cacher soigneusement leur apparition et les éliminer au plus vite pour ne pas déprécier la race ou, au contraire, doit-on en conserver un certain nombre comme meneurs, témoins de comptage ou détecteurs des carences minérales? Au cas où un certain nombre de variants colorés sont conservés, ne vont-ils pas être une source de contamination des laines blanches par des fibres pigmentées et, enfin, trouverat-on à vendre les laines naturellement colorées qu'ils vont produire?

Ces dernières années, en Australie et Nouvelle-Zélande où un élevage de moutons colorés en vue de la production lainière est en développement, ces problèmes se sont naturellement trouvés reposés (cf. SAcsos, I979; CurTis, I979; L/AUVERGNE, I980).

En France, on attache actuellement beaucoup moins d'importance à la laine, qu'elle soit colorée ou non d'ailleurs, que dans ces pays (cf. RoUGEOT, r980), mais la situation peut changer et l'on a noté récemment une demande accrue pour les laines naturellement pigmentées (LAUVERGNE et al., I979).

Aussi, après nous être intéressé à divers variants colorés de races françaises et étrangères (cf. par ex. LAUVERGNE, I96I, I969, I975; LAUVERGNE et ADALSTEINSSON, I976; LAUVERGNE et HOOGSCHAGEN, I978), avons-nous pensé qu'une étude de génétique des populations des variants colorés dans une race française blanche serait utile. Un concours de circonstances a fait qu'elle était possible en race Mérinos d'Arles grâce à la disponibilité pour enquêter sur place de l'un de nous (A. SADORGE).

Le présent article est consacré à l'étude de la génération parentale. I1 est destiné à préciser l'éventuelle présence d'animaux colorés parmi les adultes, reproducteurs ou non (I), les raisons pour lesquelles on les garde et plus généralement la répartition đes reproducteurs mâles et femelles dans les isolats génétiques que constituent les troupeaux, de même que la nature et l'intensité des échanges entre isolats en replaçant ceux-ci dans leur milieu géographique, aussi bien lors de l'hivernage qu'en estive.

Le second article sera consacré plus spécialement à la mise en évidence phénotypique et génotypique des allèles de couleur par l'analyse des données de ségrégation à l'estimation des fréquences et à l'étude de leur équilibre sous l'influence des forces de sélection de migrations, etc., mis en évidence dans le présent article.

\section{II. - Matériel et méthodes}

\section{A. - La race Mérinos d'Arles}

Le Mérinos d'Arles est une des races Mérinos qui subsistent en France. Sa création remonte au milieu du xvIII siècle, par croisements d'absorption de populations locales avec des sujets Mérinos venus d'Espagne (QuITTET, I965).

(1) Floucats : mâles castrés qui portent un ou plusieurs flots de laine épargnés lors de la tonte. Munis d'une sonnaille, ils étaient déjà utilisés aux côtés des menons (boucs castrés) pour la conduite des troupeaux dans la transhumance provençale traditionnelle (cf. LAURANS, 1976). Sont encore présents dans la plupart des troupeaux transhumants de cette région. 
Actuellement, on compte en France environ 500 ooo Mérinos d'Arles pour I50 ooo Mérinos de l'Est et Io ooo Mérinos Précoces. Les troupeaux Mérinos d'Arles, dont beaucoup transhument en été sur les pâturages alpins, sont, pour l'essentiel, cantonnés en hiver dans les Bouches-du-Rhône et le Var (Desvignes et Peyroux, r97I).

Le standard coloré de la race, dont le siège du flock-book est au Domaine $d u$ Merle à Salon-de-Provence (Bouches-du-Rhône), est le blanc pur, mais il naît de temps en temps des animaux colorés dont certains sont conservés comme floucats ou même comme brebis reproductrices, les mâles de service, même dans les troupeaux non inscrits, n'étant, quant à eux, jamais pigmentés.

\section{B. - Les troupeaux des enquêtes}

L'enquête de 1979 a porté sur 28 troupeaux Mérinos d'Arles (22 non inscrits et 6 inscrits) qui ont été passés en revue au mois de janvier I979, à la fin de l'agnelage d'automne qui se déroule entre octobre et décembre.

Les animaux colorés (agneaux et reproducteurs) ont été examinés toutes

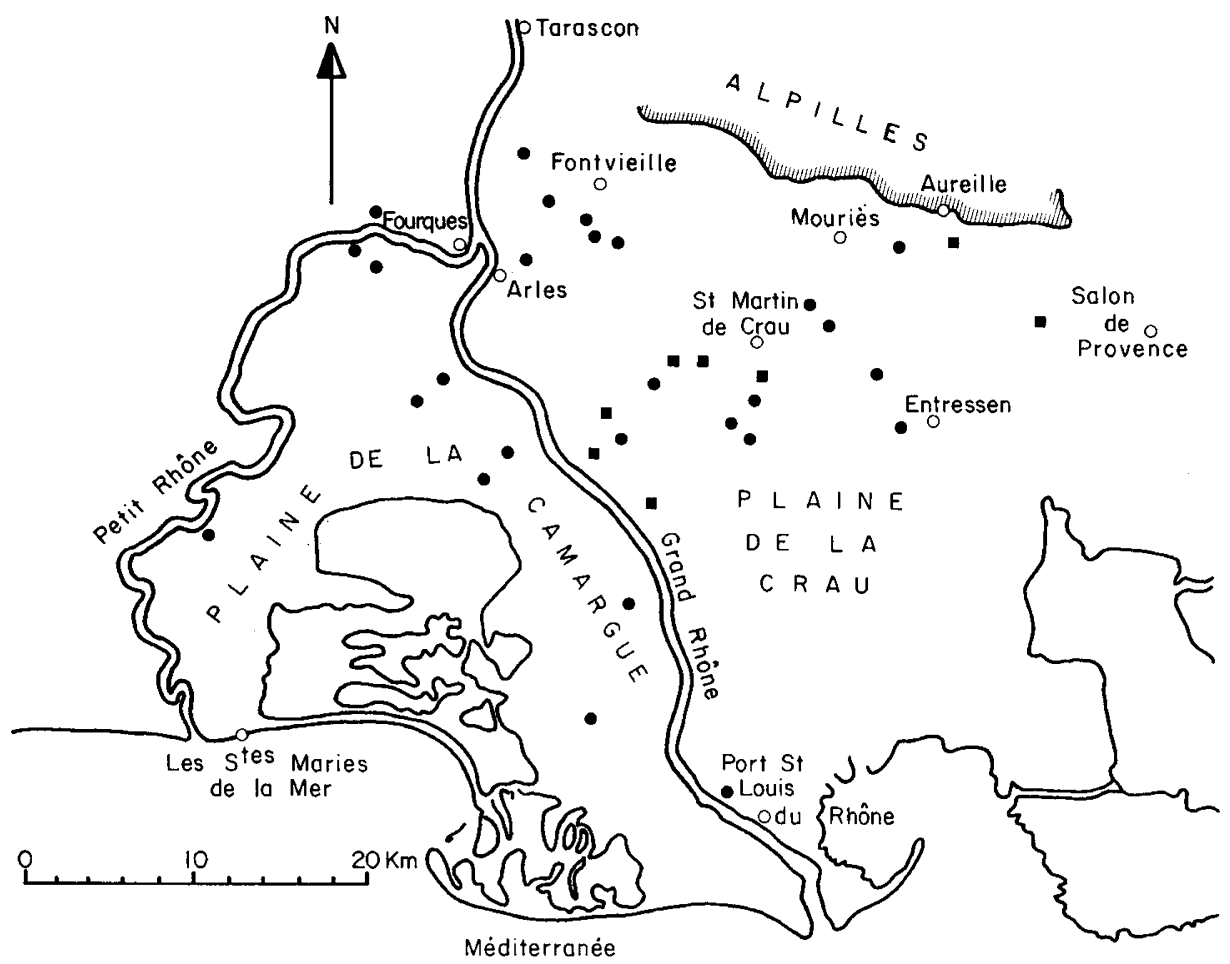

FIG. $x$. - Localisation hivernale des 35 troupeaux Mérinos d'Arles des enquêtes r979 et r980 (pour les 4 éleveurs ayant changé d'adresse en I980, on a donné la localisation de I979).

Location during the winter of the 35 Arles Merino flocks of the 1979 and I980 surveys (for the 4 breeders who moved between 79 and 80 the 79 location is given).

Troupeau inscrit Registred flock
- Troupeau non itiscrit Unregistred flock
Chef-lieu de commune Community 


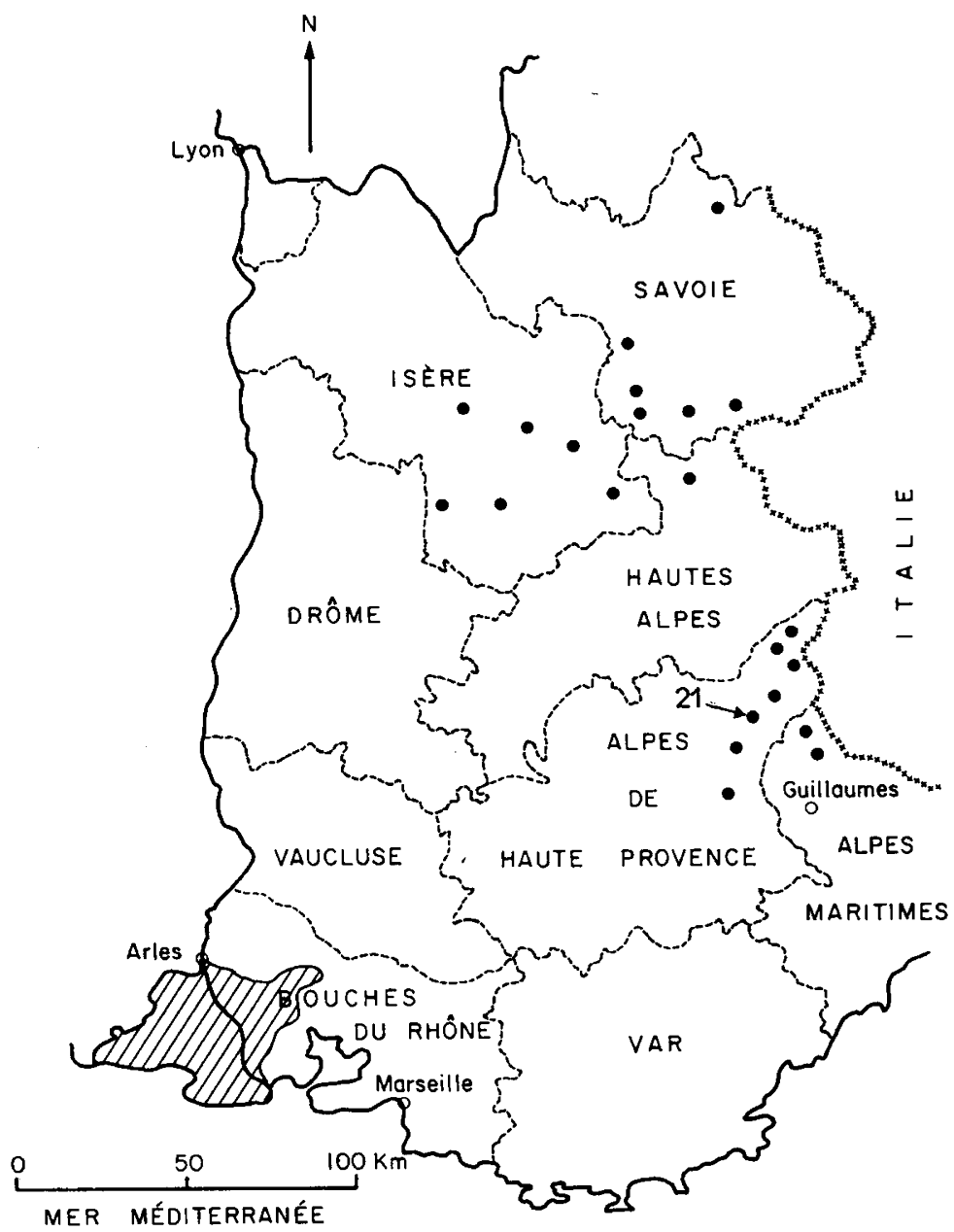

Fig. 2. - Lieu d'estivage des 22 troupeaux transhumants de l'enquête I98o.

Summer location of the 22 transhumants flocks of the r980 survey.

IN Zone d'hivernage des troupeaux

Zone of winterage of the flocks

- Localisation estivale d'un troupeau

Summer location to a flock

Cartographie : Carte administrative de la France au $I / I 400000$ de l'Institut Géographique national.

les fois qu'il a été possible de le faire et une fiche individuelle a été alors établie pour chacun d'eux.

L'enquête de I980 a été entreprise après qu'un premier dépouillement des résultats đe I979 eut montré que des renseignements complémentaires étaient nécessaires, en particulier pour estimer l'intensité des échanges de mâles, mais aussi l'intervalle de génération, les habitudes de transhumance et autres caractéristiques démographico-génétiques. 
Mouvements estivaux des 25 troupeaux Mérinos d'Arles de l'enquête 1980

Summer movements of the 25 Arles Mérino flocks of the rg8o survey

\begin{tabular}{|c|c|c|c|}
\hline Divers comportements & & $\begin{array}{c}\text { Nombre } \\
\text { de troupeaux }\end{array}$ & $\%$ \\
\hline 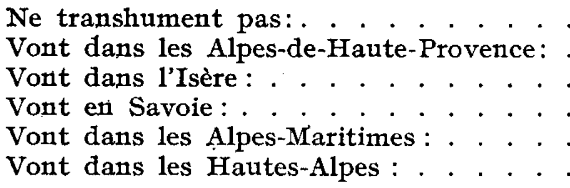 & 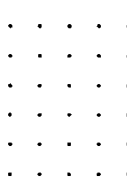 & $\begin{array}{l}3 \\
7 \\
6 \\
6 \\
2 \\
\text { I }\end{array}$ & $\begin{array}{r}12 \\
28 \\
24 \\
24 \\
8 \\
4\end{array}$ \\
\hline
\end{tabular}

Dix-huit des 28 troupeaux inventoriés en I979 ont été repris dans l'enquête de janvier I980 (n ${ }^{\text {os }} 8$ à 25 du tabl. 2, sauf le $n^{0}$ I9 bis), auxquels sont venus s'ajouter 7 nouveaux troupeaux (n 26 à 32 du tabl. 2).

Ces 35 troupeaux échantillonnés hivernent sur le territoire des communes des Saintes-Maries-de-la-Mer, d'Arles, de Fontvieille, de Tarascon, de Mouriès, d'Aureille, de Salon-de-Provence, d'Entressen et de Port-Saint-Louis-du-Rhône, qui appartiennent au département des Bouches-du-Rhône, ainsi que sur la commune voisine de Fourques (département du Gard), c'est-à-dire en Camargue et en Crau pour l'essentiel (cf. fig. I).

La plupart de ces éleveurs sont des " herbaciers ", c'est-à-dire qu'ils louent leurs pâturages d'hiver. On note donc une certaine instabilité dans leur localisation hivernale. Ainsi, parmi les r 8 éleveurs étudiés à la fois en I979 et I980, 4 (soit 22,2 p. Ioo) avaient changé de domicile dans 1'intervalle. La distance moyenne de leur déplacement $(6,7 \mathrm{~km}$ à vol d'oiseau) restait cependant faible.

En été I979, 22 sur les 25 éleveurs présents à 1'enquête I980, soit 88 p. roo d'entre eux, avaient estivé dans les Alpes (cf. tabl. I et fig. 2).

Les lieux d'estives étaient en moyenne à $220 \mathrm{~km}$ à vol d'oiseau des lieux d'hivernage.

\section{C. - Identification des phénotypes et génotypes colorés}

Un examen préalable a permis de dresser la liste des phénotypes colorés en Mérinos d'Arles. Ills seront étudiés en détail dans le second article. Pour la présente étude, il nous suffit de savoir qu'il y a le noir, le rouge et le blaireau qui peuvent aussi être panachés.

Le blaireau, appelé localement bigaillé, correspond au phénotype badger face décrit en particulier en Islande par ADALSTEINSSON (I970). Il a été repéré en Corse par LAUVERGNE et AdALSTEINSSON (I976). Le ventre et l'intérieur des pattes sont noirs, il y a des listes noires en tête, le patron est quelque peu variable.

Chez l'adulte noir, il existe un brunissement des extrémités des mèches qui donne une couleur parfois assez claire à la toison, mais la couleur originale se reconnaît sans peine, pour peu que l'on ouvre celle-ci. 


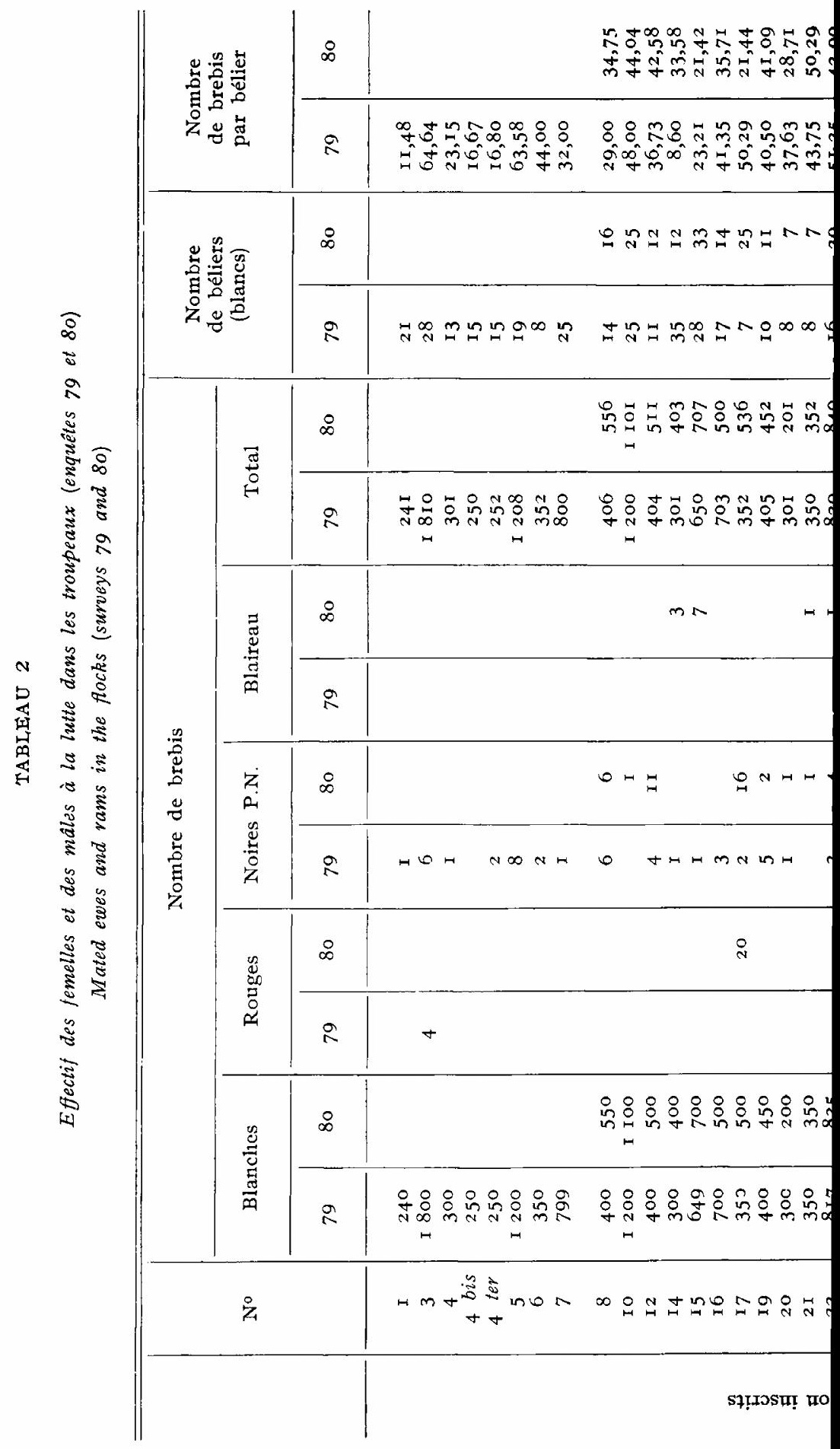




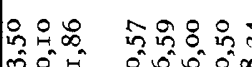

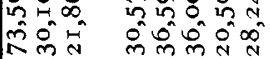

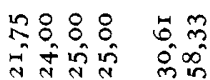

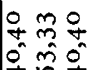

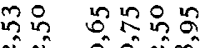

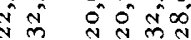

\begin{tabular}{|c|c|c|c|c|c|c|c|}
\hline In & & $\stackrel{\infty}{m}$ & $\begin{array}{l}\vec{\infty} \\
\text { مी } \\
\hat{n}\end{array}$ & 움움 & $\hat{H}$ : & & $\overbrace{i=1}^{n} \hat{i}$ \\
\hline 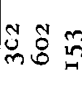 & 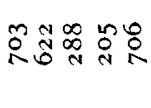 & $\frac{0}{+}$ & $\begin{array}{l}0 . \\
\hat{A} \\
\text { in }\end{array}$ & & moo & $\stackrel{\circ}{\circ}$ & 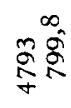 \\
\hline
\end{tabular}

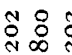

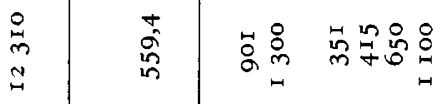

Nิ

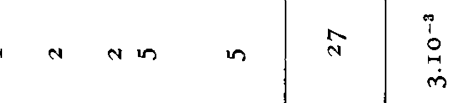

$-\mid \begin{array}{r}i \\ 0 \\ 0 \\ 0\end{array}$

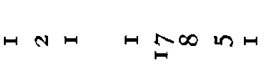

$\gtrless \quad$\begin{tabular}{l|l}
$i$ \\
0 \\
$\infty$
\end{tabular}

\begin{tabular}{l|l|ll}
$\circ$ & $i$ & $H$ & $H$
\end{tabular}

(

용

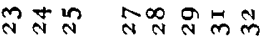

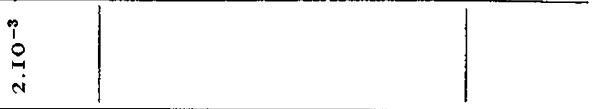

ì

옹영 웡웡요

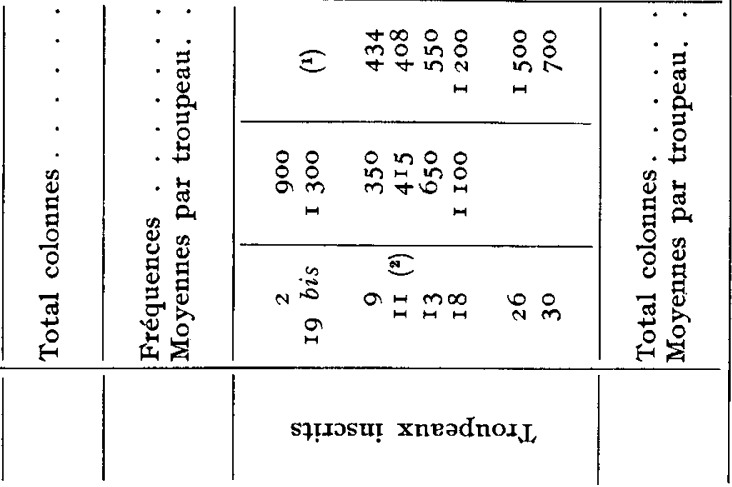


Chez les adultes rouges, la couleur se retrouve uniquement dans les parties poilues (tête et pattes), le pigment rouge présent à la naissance disparaît en effet rapidement de la toison.

\section{D. - Étude démographique}

La distribution de la taille des troupeaux femelles aussi bien que celle des effectifs mâles de service a été analysée par un test de log normalité.

On a essayé de rechercher la relation entre le nombre de reproducteurs mâles et celui des femelles.

La prolificité des brebis agnelants lors des deux agnelages envisagés a également été précisée.

On a analysé ensuite la fréquence des adultes colorés de manière à voir s'il $\mathrm{y}$ avait un rapport de leur nombre avec la taille du troupeau et plus généralement pour savoir quelles étaient les motivations de leur conservation dans les troupeaux.

Le processus de migration du matériel génétique entre les troupeaux a été abordé par les biais des échanges de béliers entre troupeaux.

\section{III. - Résultats}

\section{A. - Les effectits des reproducteurs}

Dans le tableau 2 sont donnés les effectifs approximatifs des brebis de divers phénotypes colorés dans les différents troupeaux inventoriés en I979 (agnelage automne 78) et en I 980 (agnelage automne 79), ainsi que celui des mâles de service (tous blancs, rappelons-le) utilisés. Dans le tableau 3 sont données les moyennes des effectifs (ainsi que le nombre de brebis par bélier à la lutte).

\section{B. - La loi de distribution des troupeaux selon leur taille}

Elle affecte une forme dissymétrique aussi bien pour les troupeaux inscrits que pour les troupeaux non inscrits (cf. fig. 3).

Le test graphique de log normalité fait sur les seuls troupeaux non inscrits pour les mâles et les femelles (les troupeaux inscrits étant trop peu nombreux) est donné dans la figure 4 .

\section{C. - Le sex-ratio à la lutte}

Dans le tableau 3 (déjà cité) on trouvera le nombre moyen de brebis par bélier dans les différents types de troupeau pour les différentes années.

Dans la figure 5 on a porté en abscisses la taille du troupeau femelles non inscrites lors de la lutte de printemps r979 et de la lutte I980 pour les troupeaux non déjà présents dans l'enquête I979 et en ordonnée le nombre de mâles correspondants.

La disposition du nuage de points tend assez vain le calcul d'un coefficient de corrélation. 
MÉRINOS D'ARLES COLORÉS I

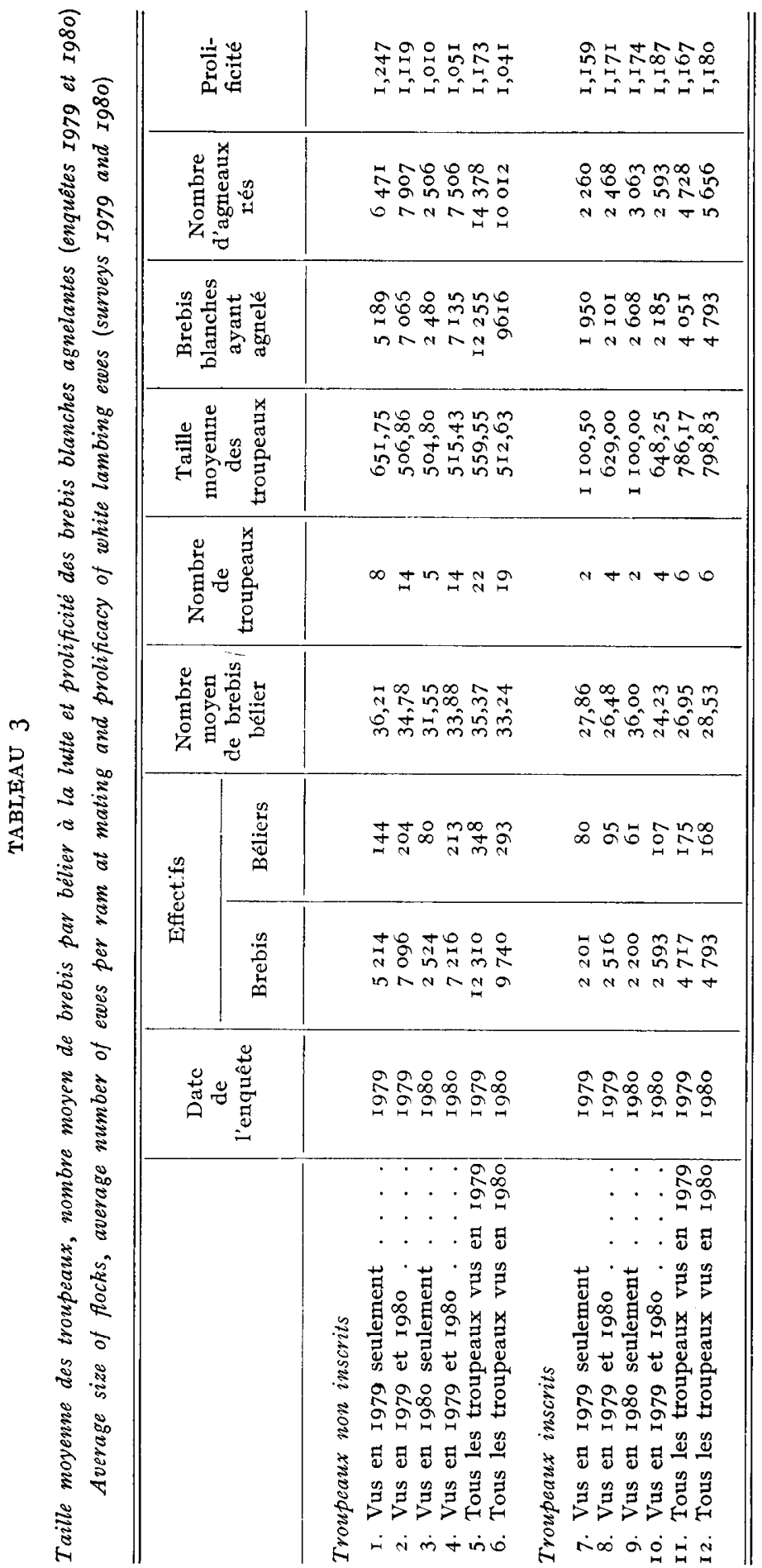



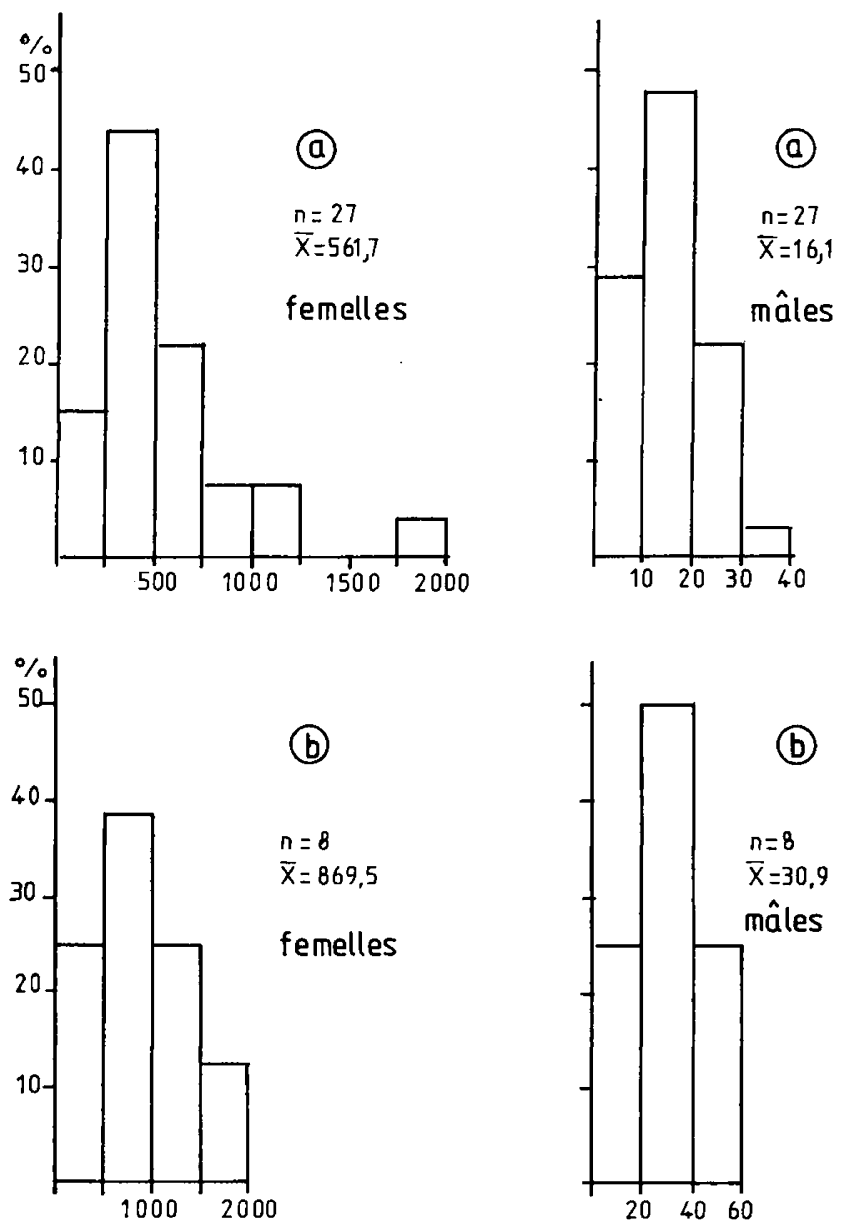

FIG. 3. - Distribution des troupeaux des enquêtes 79 et 80 (effectifs des femelles et des mâles) selon leur taille. On a pris la moyenne 79/80 pour les troupeaux présents les 2 années.

a) Troupeaux non inscrits.

b) Troupeaux inscrits.

Distribution of the flocks of 79 and 80 surveys according their size (erves and rams). The average size has been taken for the flocks which figure both years.

a) Non registered flocks.

b) Registered flocks.

Dans la figure 6 on a caractérisé les mêmes troupeaux dans le plan effectif des troupeaux femelles/nombre de femelles par mâle à la lutte.

\section{D. - Prolificité des brebis}

Dans le tableau 3 on a également reporté les effectifs globaux des naissances qui seront donnés en détail dans l'article suivant, de manière à déterminer la prolificité des brebis lors des agnelages d'automne 78 et 79 . 


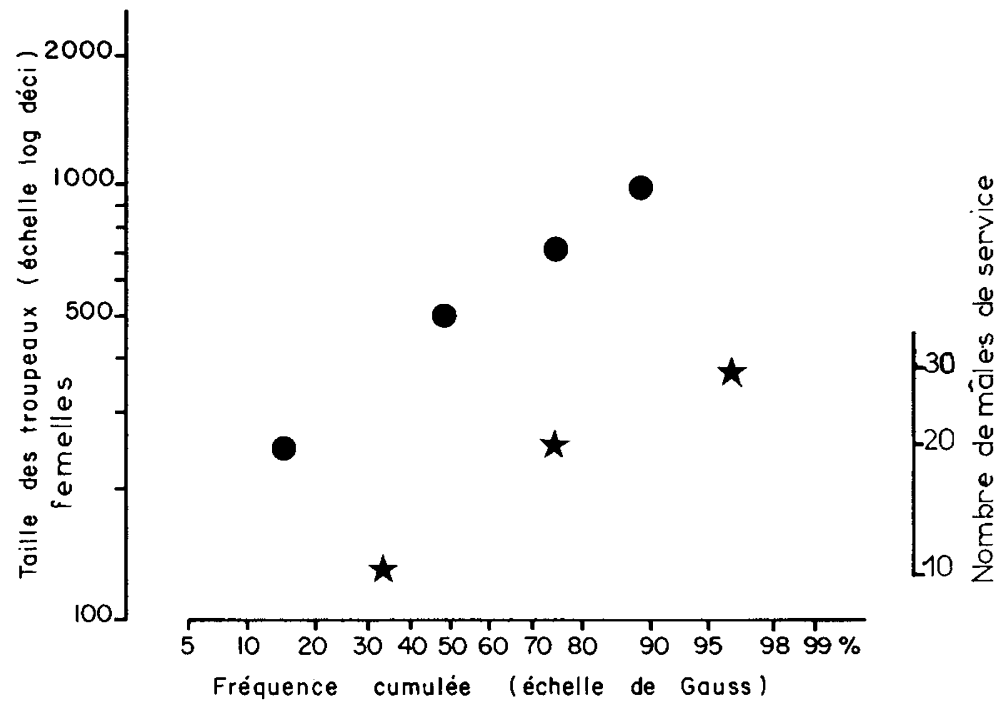

FIG. 4. - Test de log normalité de la loi de distribution de la taille des troupeaux Mérinos d'Arles non inscrits.

Test of a log normal distribution for the flock size of unvegistered Arles Merino flocks.

- Femelles $\quad \star$ Mâles

Females Males

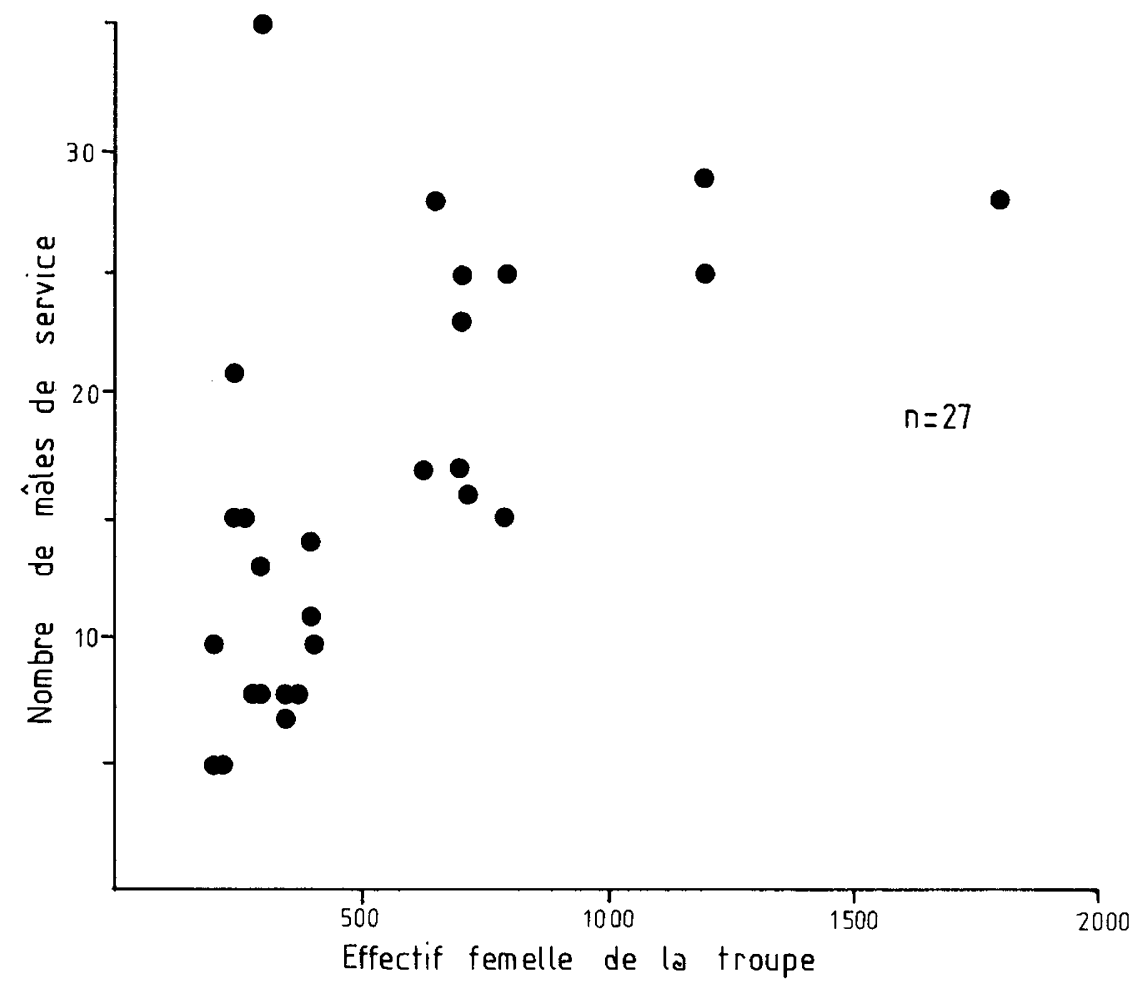

FIG. 5. - Corrélation entre l'effectif temelle des troupeaux et le nombre de males de service.

Correlation between the size of the ewe flock and the number of used males. 


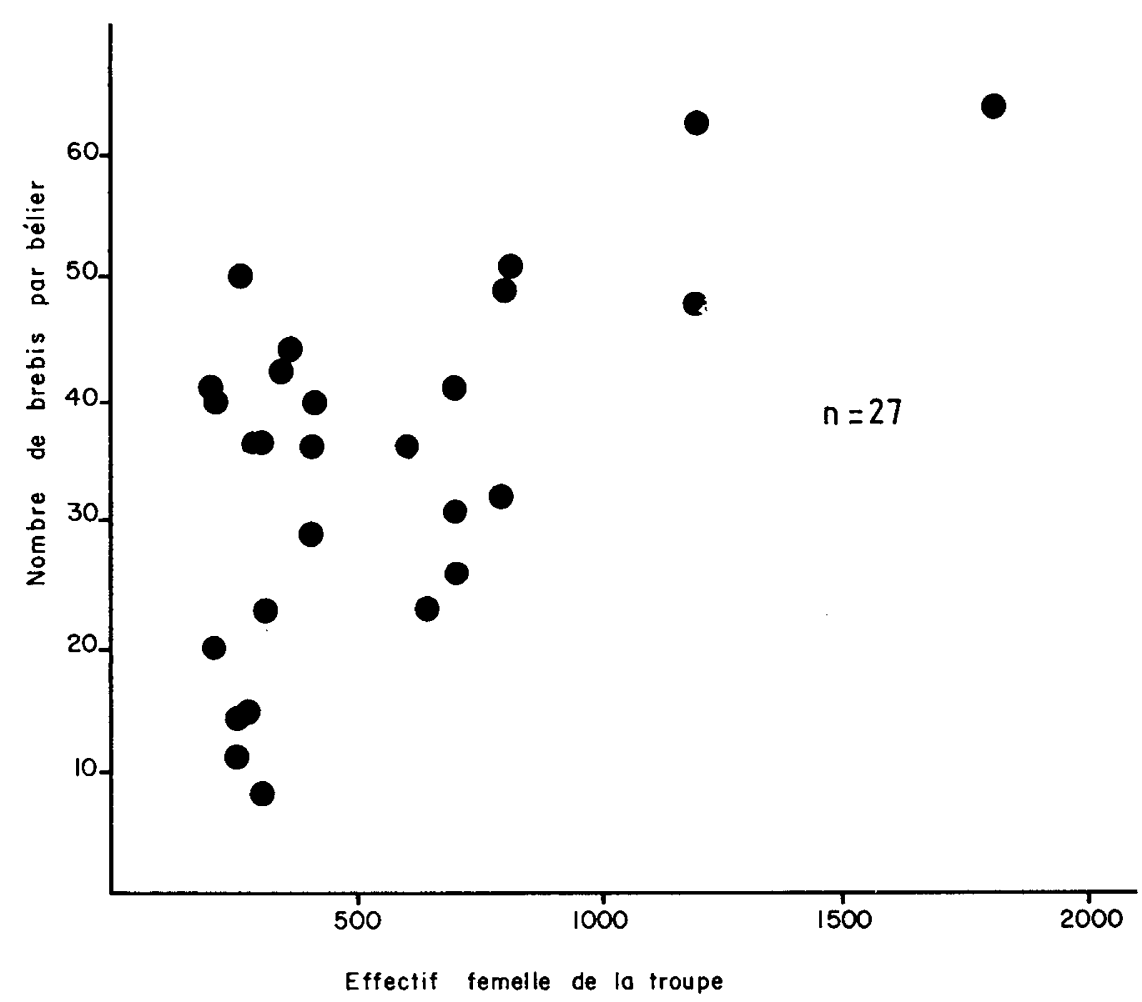

FIG. 6. - Corrélation entre l'effecrif femelle du troupeau et le nombre de femelles par bélier.

Correlation between the size of the ewe flock and the number of ewes per ram.

\section{E. - Mouvement des reproducteurs mâles}

La provenance des mâles utilisés pour la lutte de printemps 1979 est donnée dans le tableau 4. Le tableau 5 résume les données du tableau 4.

\section{F. - Les floucats de diverses couleurs}

La répartition des floucats de diverses couleurs dans les troupeaux de l'enquête r980 est donnée dans le tableau 6.

\section{G. - Les fréquences comparées des floucats et des brebis de diverses couleurs}

Dans le tableau 7 on a présenté des paramètres qu'il est possible d'extraire des tableaux 2 et 6 : pourcentage des troupeaux avec floucats, avec floucats colorés, avec brebis colorées, nombre de brebis par floucat, fréquence des floucats et des brebis colorés dans les troupeaux non inscrits et inscrits. 
TABLEAU 4

Provenance des béliers utilisés pour la lutte 1979 (enquête 1980)

Origin of the rams used for the 79 mating ( 1980 survey)

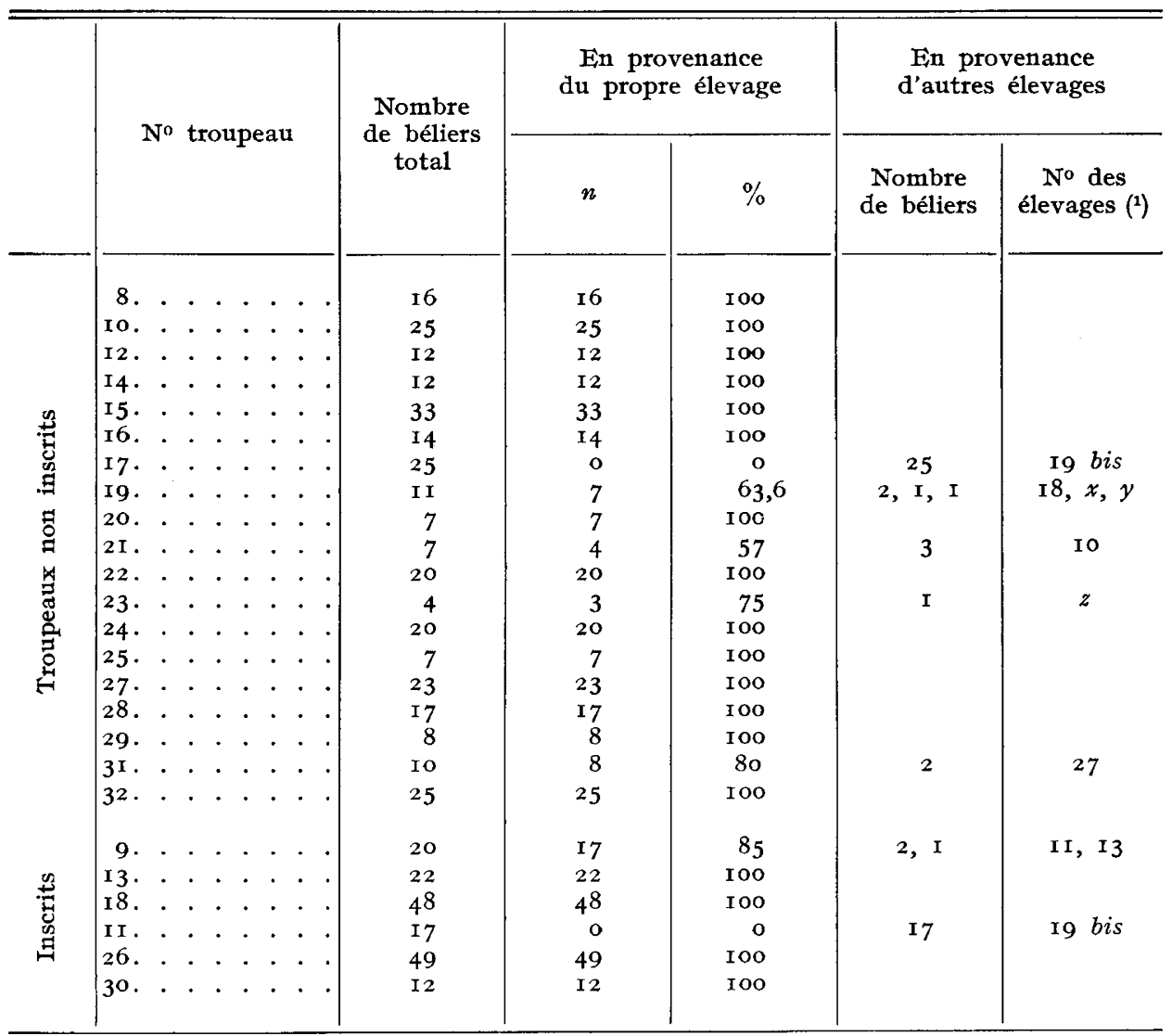

(1) Les numéros de troupeau renvoient à la liste du tableau 3 .

rg bis est le troupeau du Merle, avec son haras de béliers.

$x, y$ et $z$ sont des troupeaux non inscrits non envisagés dans l'enquête.

H. - Liaison du nombre d'adultes colorés avec la taille des troupeaux

Les corrélations entre le nombre d'animaux colorés, le nombre de floucats et 1'effectif des troupeaux de l'enquête I980 sont données dans le tableau 8 .

La liaison entre le nombre de brebis colorées et la taille des troupeaux non inscrits de l'enquête I980 est illustrée dans la figure 7 .

\section{I. - Age des béliers}

L’âge moyen des béliers utilisés en 1979 était de 3,3 ans lors de la lutte. 
TABLEAU 5

Paramètres concernant la provenance des béliers

Parameters for the origin of the rams

\begin{tabular}{|c|c|c|}
\hline Rubriques & $\begin{array}{l}\text { Troupeaux } \\
\text { non inscrits }\end{array}$ & $\begin{array}{l}\text { Troupeaux } \\
\text { inscrits }\end{array}$ \\
\hline $\begin{array}{l}\text { Nombre total de troupeaux } \\
\text { Nombre de troupeaux n'utilisant que leurs propres } \\
\text { béliers. } \\
\text { Pourcentage } \\
\end{array}$ & $\begin{array}{l}19 \\
14 \\
73,68\end{array}$ & $\begin{array}{l}6 \\
46,60\end{array}$ \\
\hline 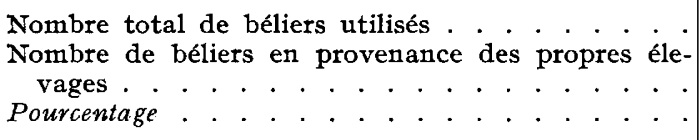 & $\begin{array}{l}296 \\
26 \mathrm{I} \\
88,17\end{array}$ & $\begin{array}{l}\mathbf{1} 68 \\
\mathbf{1} 48 \\
88,09\end{array}$ \\
\hline $\begin{array}{l}\text { Nombre de béliers étrangers utilisés dans les trou- } \\
\text { peaux . de béliers étrangers en provenance d'éle- } \\
\text { Nombre de } \\
\text { vages inscrits. } \\
\text { Pourcentage. } . .\end{array}$ & $\begin{array}{l}35 \\
25 \\
7 I, 43\end{array}$ & $\begin{array}{l}20 \\
20 \\
100,00\end{array}$ \\
\hline 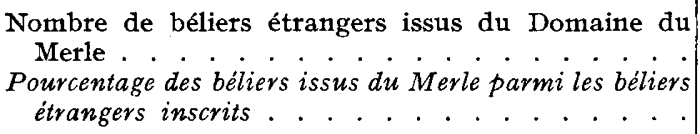 & $\begin{array}{c}25 \\
100,00\end{array}$ & $\begin{array}{l}17 \\
85,00\end{array}$ \\
\hline
\end{tabular}

\section{IV. - Discussion}

\section{A. - La précision des comptages}

Les effectifs donnés par les éleveurs, en particulier pour les brebis et les agneaux, apparaissent presque tout le temps très arrondis et même assez variables d'une année sur l'autre (cf. tab1. 2), ce qui pourrait cacher de graves imprécisions.

Ces craintes ne semblent pas justifiées. En effet :

- l'enquête a été faite par une même personne (A. SADORGE) qui connaît bien et les éleveurs et les élevages, étant éleveur lui-même. Elle a pu voir que les chiffres étaient effectivement arrondis mais non biaisés;

- les variations d'effectifs d'une année à l'autre sont dues à la condition même des éleveurs qui, rappelons-le, sont des herbaciers, c'est-à-dire doivent louer leurs pacages aussi bien en hiver qu'en été. On voit par ailleurs que ces variations individuelles se compensent : 7096 brebis en I979, 7216 en I980 par exemple pour les $\mathrm{I} 7$ élevages non inscrits qui reviennent dans les deux enquêtes (cf. tab1. 2). 
MÊRINOS D'ARLES COLORÉS I

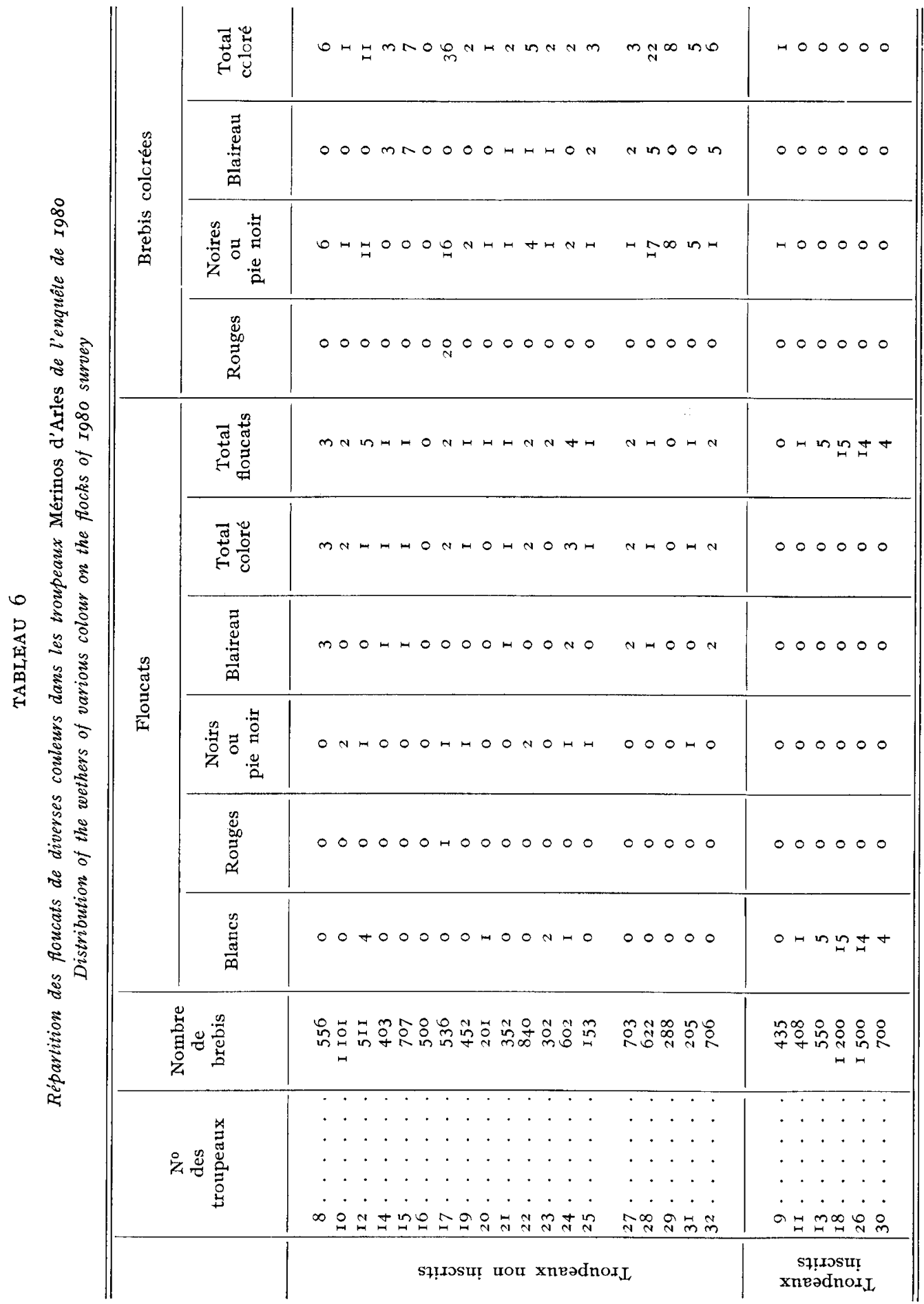




\section{TABLEAU 7}

Fréquence des floucats et des brebis colorées dans les troupeaux inscrits et non inscrits (enquête de I980: agnelage d'automne 79)

Frequencies of wethers and of coloured ewes in registered and non registered flocks ( 1980 survey)

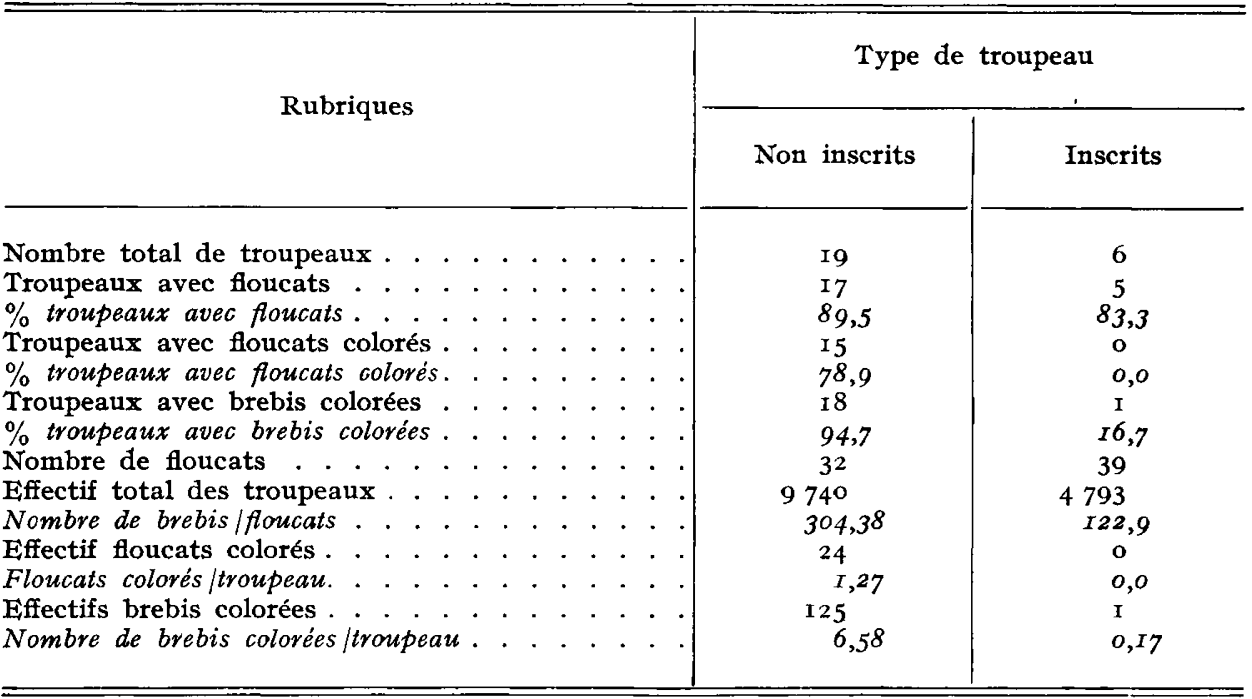

\section{TABLEAU 8}

Correlation entre les effectifs des floucats, des animaux colorés et la taille des troupeaux (enquête de Ig8o)

Correlation between the number of wethers, the number of coloured animals and flock size (rg8o survey)

\begin{tabular}{|c|c|c|c|c|}
\hline \multirow{2}{*}{ No } & \multicolumn{2}{|c|}{ Variables en corrélation } & \multicolumn{2}{|c|}{$\gamma_{x y}$} \\
\hline & $x$ & $y$ & $\begin{array}{l}\text { Troupeaux } \\
\text { non inscrits }\end{array}$ & $\begin{array}{l}\text { Troupeaux } \\
\text { inscrits }\end{array}$ \\
\hline I & Taille du troupeau & $\begin{array}{l}\text { Nombre de brebis colorées dans } \\
\text { le troupeau }\left(^{(}\right) .\end{array}$ & 0, I I & $-0,40$ \\
\hline 2 & Taille du troupeau & 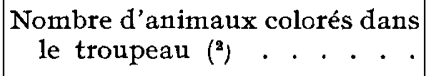 & O,I 9 & $-0,4^{\circ}$ \\
\hline 3 & Taille du troupeau & $\begin{array}{l}\text { Nombre de floucats colorés dans } \\
\text { le troupeau . . . . . . . }\end{array}$ & 0,56 & 0,00 \\
\hline 4 & Taille du troupeau & $\begin{array}{l}\text { Nombre total de floucats dans } \\
\text { le troupeau . . . . . . . . }\end{array}$ & $0,3 \mathrm{I}$ & 0,95 \\
\hline
\end{tabular}

(1) Noires, pie noir ou blaireau.

(2) Brebis et floucats. 


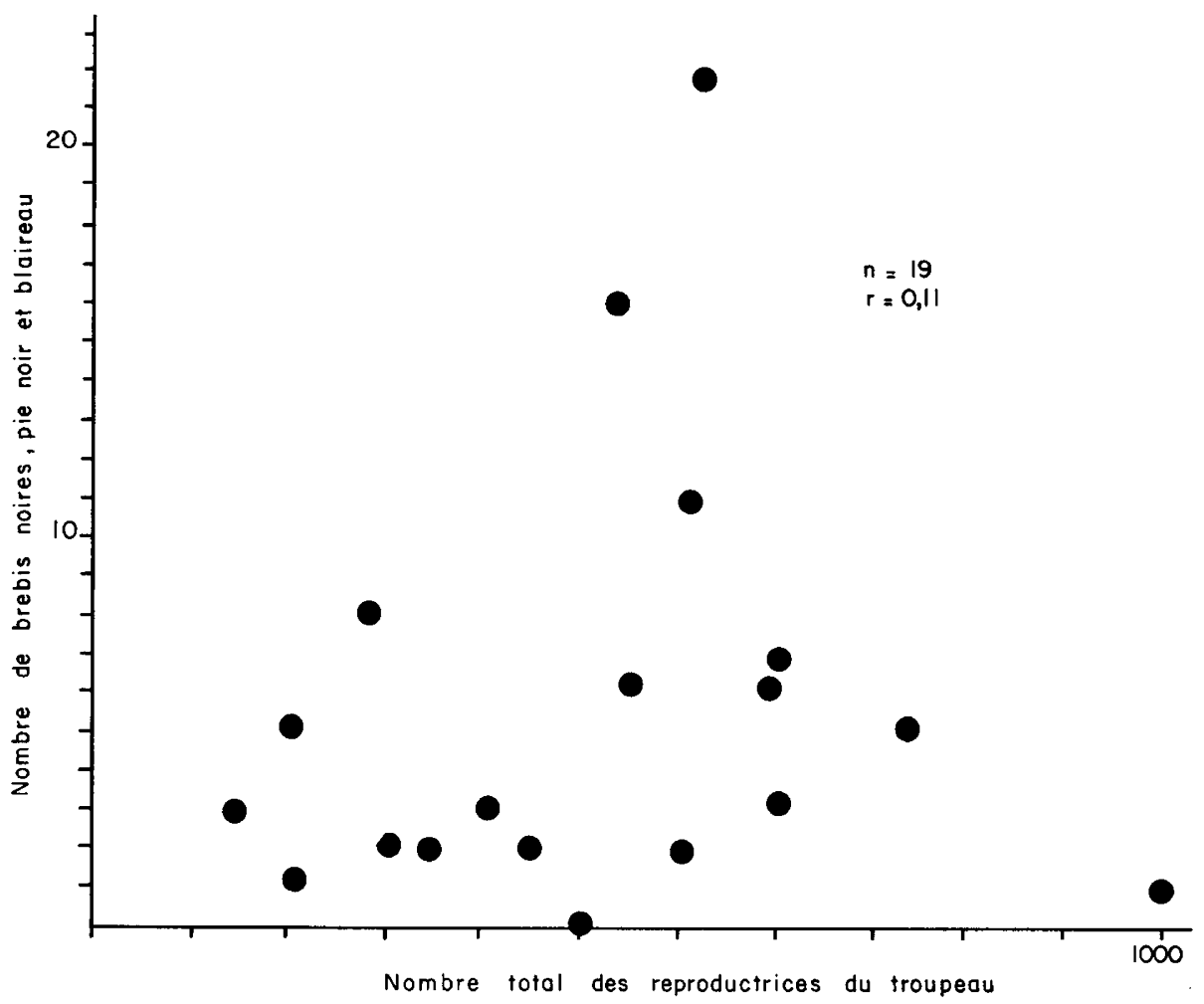

FIG. 7. - Liaison entre le nombre des brebis noires, pie noir ou blaireau dans un troupeau et la taille du troupeau. Troupeaux non inscrits, enquête de 1980 .

Number of black, piebald and badger face ewes in a flock and size of the flock. Unvegistred flocks, I980 survey.

Par ailleurs, autre vérification a posteriori, les paramètres calculables à partir des effectifs adultes et naissants paraissent tout à fait dans les normes, spécialement le nombre de brebis par bélier ou la prolificité des mères. Il en est de même, comme nous le verrons plus loin, pour la distribution des troupeaux selon leur effectif.

\section{B. - Les caractéristiques démographiques des troupeaux de l'enquête}

\section{I. - Effectifs moyens}

L'effectif femelle moyen des troupeaux non inscrits est nettement inférieur à celui des troupeaux inscrits (resp. 560 et 5 I 3 pour I979 et I980, comparés à 786 et 800 , soit une différence de 226 à 287 brebis - 28 à 35 p. Ioo en moins).

La situation n'est pas la même pour les élevages laitiers de Sardaigne que nous avions étudiés il y a quelques années, les effectifs des élevages inscrits étant inférieurs de 50 p. Ioo à ceux des non inscrits, les rendements laitiers bien supérieurs (LAUVERGNE et al., I973) compensant et au delà l'infériorité numérique. 
Pour le Mérinos d'Arles, où la production de viande est la principale spéculation et où les prolificités sont comparables de troupeau inscrit à troupeau non inscrit, les éleveurs inscrits pourraient se distinguer des non inscrits par leur meilleure aptitude à " tenir " des effectifs plus importants. Il se pourrait aussi que les éleveurs non inscrits n'aient pas fait état dans l'enquête de troupeaux annexes qu'ils peuvent avoir en association avec d'autres. La chose demande vérification.

\section{2. - Loi de distribution de la taille des troupeaux}

La figure 4 montre que l'ajustement à une loi log normale des histogrammes de distribution des troupeaux selon les effectifs mâles et femelles à la lutte est bon.

Cette loi de distribution est observée dans d'autres races, aussi bien en Sardaigne qu'en Grande-Bretagne (cf. LAUVERGNE et al., r973, qui ont testé la lognormalité des races anglaises étudiées par WIENER, I96I).

\section{3. - La prolificité des brebis}

Les prolificités relevées pour les agnelages d'automne 78 et 79 varient entre I,04 (troupeaux non inscrits en I979) et I,I 8 (pour les troupeaux inscrits en I980) (tabl. 4). Ces chiffres concernent les brebis agnelantes. Ils sont tout à fait comparables aux performances observées dans la région (par ex. au chiffre de I,I I agneau communiqué par le Syndicat ovin de Salon-de-Provence en I979).

\section{4. - Le sex-ratio à la lutte}

Il vaut en effet mieux employer cette périphrase que le terme de lot de lutte car, en zone Mérinos d'Arles, la lutte ne se fait pas en main : les béliers sont lâchés tous ensemble dans le troupeau femelle.

L'examen des figures 4 et 5 montre, comme déjà évoqué, qu'une relation régulière entre la taille des troupeaux femelles et l'effectif des mâles de service est difficile à mettre en évidence, même si en moyenne on retrouve les chiffres avancés par PRUD'HON en I97I $(32,4$ alors que nos valeurs moyennes extrêmes sont 27 et 35,4 , cf. tab1. 3 ).

En fait, le nombre de mâles apparaît proportionnel à celui des femelles d'après la figure 5 si 1'on fait abstraction du nuage de points situé en haut à gauche. De même, dans la figure 5 apparaît bien une relation régulière, bien que curvilinéaire, entre la taille des troupeaux et le nombre de brebis à la lutte, cet effectif étant en décroissance à mesure que la taille du troupeau augmente, ce qui est compréhensible car, pour les petits troupeaux, il est difficile de descendre en dessous d'un nombre minimum de béliers; on l'avait vu d'ailleurs avec les races anglaises (WIENER, I96r). Toutefois, pour que cette relation soit nette, il faut là aussi faire abstraction d'un nuage de points en haut à gauche. C'est dire que l'on est en présence d'au moins deux populations d'éleveurs quant à l'organisation de la lutte de printemps. En fait, le problème est peut-être même plus compliqué, car la lutte de printemps n'est qu'une des étapes du cycle de production annuelle, voire bisannuelle, des éleveurs de Mérinos d'Arles. Il se peut aussi qu'une partie des éleveurs utilisent systématiquement plus de mâles qu'il n'en est besoin, pour 
des raisons de sécurité, parce qu'ils y mêlent des mâles jeunes en lesquels ils n'ont pas trop confiance ou simplement par laisser-aller. Une étude plus détaillée serait nécessaire mais elle sort nettement du cadre de notre étude qui s'intéresse à ce paramètre afin de préciser la taille des isolats et leur distribution avec une mention toute particulière au sexe le moins nombreux, donc déterminant les mâles.

\section{5. - L'intervalle entre générations}

La seule mesure possible de ce paramètre, à l'aide de nos données, l'est chez les mâles avec l'âge des béliers à la mise à la lutte : 3,3 ans. Au Merle, Prud'hon, en I97I, notait un intervalle de génération de 3,49 années.

Cet âge moyen est aussi à rapprocher des chiffres de 3,I à 3,9 observés en Sardaigne (LAUVERGNE et al., déjà cités).

\section{6. - Le système d'échange des béliers}

Nos données révèlent (tab1. 4 et 5 ) un pourcentage élevé de béliers en provenance du propre troupeau des éleveurs ( $88 \mathrm{p}$. Ioo), aussi bien pour les troupeaux non inscrits qu'inscrits d'ailleurs.

Cette tendance est générale dans 1'élevage ovin européen, mais elle n'atteint ce degré d'intensité ni parmi les races anglaises étudiées par WIENER (I96I) (au maximum 40 p. IOo), ni en race Sarde où cette proportion était de $34 \mathrm{p}$. Ioo pour les élevages inscrits et de $58 \mathrm{p}$. Ioo pour les élevages non inscrits.

Peu d'auteurs semblent avoir étudié la motivation de ce phénomène. En Angleterre, cette proportion de béliers autochtones est dans une certaine mesure en rapport avec la taille moyenne des troupeaux d'une race donnée, les races à petits effectifs moyens ayant une plus grande tendance à l'exogamie, car sans doute la consanguinité y est plus menaçante. Mais le phénomène ne semble pas jouer lorsque les tailles atteignent plusieurs centaines de bêtes, cas où, effectivement, la consanguinité est moins à redouter vu le nombre des béliers employés.

En ce qui concerne le Mérinos d'Arles, le progrès génétique pâtit sans doute de cette tendance endogame. On peut en effet voir que les béliers sélectionnés par le Flock-book et entretenus dans le haras du Domaine du Merle ne constituent que 7,5 p. roo des béliers utilisés dans les troupeaux non inscrits (tab1. 5).

En fait la tendance endogame que nous notons au niveau du choix des mâles peut être contrecarrée par des échanges et achats de femelles que l'on peut dans une certaine mesure subodorer d'après les variations parfois notables d'effectifs d'une année à l'autre (cf. tabl. 2). et par des saillies incontrôlées faites par des béliers étrangers au cours de l'estive. Ainsi s'explique par exemple la présence de brebis rouges dans l'élevage $\mathrm{n}^{\circ}$ I7 en I980 (cf. tab1. 2) et la soudaine apparition d'un lot d'agneaux rouges dans l'élevage $n^{0} 2$ I (I). Toutefois, cette dernière éventualité est sans doute de portée restreinte car les troupeaux estivent en principe une fois la lutte de printemps achevée, surtout les troupeaux inscrits.

(1) D'après des données présentées dans le $2^{\mathrm{e}}$ article de la série, cf. aussi la figure 2 qui montre la localisation de ce troupeau en estive, proche de la zone du Mourerous centrée sur Guillaumes. 


\section{C. - L'utilisation des animaux adultes colorés et des castrats dans les troupeaux}

Nous rappelons tout d'abord que ces adultes colorés peuvent être des femelles ou des mâles castrés (tab1. 2, 6 et 7) et que les troupeaux inscrits ne conservent pratiquement aucun adulte coloré. En tout état de cause même dans les troupeaux non inscrits les fréquences sont faibles : 0,4 p. Ioo en I978, I,2 p. Ioo en I979 pour les brebis (tabl. 2), cinq fois moins pour les floucats colorés en I979 (tabl. 7).

Quant à la motivation de cette conservation, on pourrait penser tout d'abord à la pratique du comptage rapide en alpage qui pourrait se combiner avec le guidage si les floucats étaient colorés.

Si la première utilisation (de comptage) prévalait, il devrait y avoir une corrélation étroite entre le nombre total d'animaux colorés et l'effectif des troupeaux, alors que pour la seconde la liaison devrait être nette entre le nombre des floucats colorés et l'effectif des troupeaux.

La première liaison est faible ou inexistante : $r=0,19$ pour les troupeaux non inscrits, $r=-0,40$ pour les troupeaux inscrits (tabl. 8, ligne 2). En fait, l'examen du nuage de points de la figure 7 montre que - peut-être comme pour le sex-ratio à la lutte - on est en présence de deux populations d'éleveurs : certains qui élèvent en gros d'autant plus de brebis noires qu'ils ont d'animaux et d'autres qui ne tiennent pas compte de cette ratio. Là encore le coefficient de corrélation est d'un secours limité pour suivre le phénomène.

La corrélation entre le nombre de floucats colorés et la taille des troupeaux non inscrits est plus nette, $r=0,58$ (tabl. 8, ligne 3), mais la liaison beaucoup plus forte entre la taille des troupeaux et le nombre total de floucats, tous blancs dans les troupeaux inscrits $(r=0,95:$ tab1. 8 , ligne 4$)$, indique que la couleur ne joue qu'un rôle secondaire en la matière : on conserve des floucats (colorés ou non, peu importe) en tant que meneurs.

Ainsi les animaux colorés conservés dans les troupeaux non inscrits n'ont guère d'utilité pratique objectivement décelable à 1'heure actuelle, vu que la laine colorée se vend plutôt plus difficilement que la laine blanche. On doit donc rechercher des raisons traditionnelles que les éleveurs ont d'ailleurs parfois évoquées devant nous : habitudes ancestrales, rôle de talisman, de décoration, etc.

\section{V. - Conclusions}

L'étude de la génération parentale montre une population où les méthodes d'élevage, en particulier la pratique de la lutte, varient grandement entre troupeaux. L'endogamie de chaque troupeau est également notablement élevée et mériterait d'être mise en rapport plus étroit avec le système d'élevage. En ce qui concerne la conservation d'adultes (reproducteurs ou non) colorés, on note également des comportements divers qui, à l'échelon de la population, contribuent à maintenir une fréquence, faible il est vrai mais assez constante, d'animaux colorés. Les motivations de telles attitudes restent également à être précisées.

Au total, une telle étude, justifiée par la carence des connaissances en la matière, est encore fort imparfaite et soulève beaucoup plus de problèmes qu'elle n'en résout. 


\section{Remerciements}

Les commentaires des lecteurs du manuscrit Ph. DREUx (E.N.S. Zoologie), Ph. Mterat (I.N.R.A., Génétique animale) et M. PRUD'HoN (E.N.S.A. Montpellier) nous ont été d'un précieux secours pour l'élaboration de cet article.

\section{Summary}

\section{The color variants in the Arles Merino sheep. I. Studies in the parent population}

Surveys of 1978 and 1979 lambing made resp. in 1979 and 1980 were performed among 35 Arles Merino flocks: 8 registred, 27 tion registred. These flocks winter in Crau and Camargue plains and spend the summer in the Alpes. This is a preliminary study of the parent population to a mendelian and population genetics study.

The average size of genetic isolates (the flocks) varies between 786 and 800 ewes for the registred flocks and 513 and 560 ewes for the unregistred ones in resp 1978 and 1979 . The corresponding average numbers of males are, 29, 28, I6 and 16.

The distribution law for the size of female and male unregistered isolates is $\log$ normal.

There is an average of 27 to 28.5 ewes for ram at mating in registered flocks and 33 to 35.4 for the unregistered ones. The linkage between this parameter and the size of flocks is not regular, this could indicate a kind of diversity in the behaviour of breeders. At automn lambing the prolificacy of ewes was between I.04 and I.I 8 lamb for lambing ewe.

The exchange of genetical material for the male sex is restricted: only i $2 \mathrm{p}$. I oo for both registered and unregistered flocks, even if the flocks are geographically quite close from each other during the wintering, when the mating is performed. Some uncontrolled migrations of genes may be done during the summertime when Merino flocks are mixed with other flocks on summer pastures.

In registered flocks only white coloured reproducers are accepted but 3 ewes. In unregistered flocks if all the males are white there are some coloured ewes: red (.03 and .02 p. I0o in resp. 78 and 79), black or black piebald (resp. .4 and .8 p. 100), or badger face (resp. .oo 8 and $.3 \mathrm{p}$. 100). The grand total of coloured ewes was $.4 \mathrm{p}$. roo in 1978 and $1.2 \mathrm{p}$. Ioo in r979. In these unregistered flocks there are, moreover, some wethers (called floucats) which may be coloured: $3 \mathrm{p}$. roo at the r 980 survey.

Coloured ewes or wethers are obviously not kept for the use of counting the flocks in the field. It looks like the wethers are used as leaders of the flocks, specially in the registered flocks but without taking account if they are coloured or not. Therefore one thinks that keeping some coloured adults is an heritage of tradition.

\section{Références bibliographiques}

Adalsteinsson S., I970. Colour inheritance in Icelandic sheep and relation between colour, fertility and fertilization. $J$. Agr. Res. Icel., 2, 3-135.

CURTIS C., 1979. The black and coloured wool industry in Australia. Australian Wool Corporation, Melbourne, Australia, 27 p. Ronéoté.

Desvignes A., PEyroux B. DU, r97I. Les races Mérinos, in Les principales races de l'Élevage Français, Cofranimex, Paris, fasc. de $8 \mathrm{p}$.

LAAURANS R., I976. Les animaux meneurs de troupeaux ovins transhumants du Midi méditerranéen. I er Coll. Ethnosci. Paris, nov. 1976. Institut international d'Ethnosciences, Paris, 50.

LAUVERGNE J. J., I96r. Sur le déterminisme génétique de la couleur noire dans la race $B l e u$ du Maine. Ann. Génét. (Sem. Hópit.), 2, 47-52.

LAUVERGNE J. J., I969. Hérédité de la couleur blanche du mouton Berrichon eroisé à des Solognots. Ann. Gén. Sél. Anim., 1, 219-226. 
LAUVERGNe J. J., I975. Génétique de la couleur de la toison de trois races ovines françaises : Berrichone, Bizet et Solognote. Ann. Génét. Sél. Anim., 7, 263-276.

LAUVERGNE J. J., 1980. Le petit élevage đe moutons colorés en Australie. Ethnozootechnie (26), 65-7I.

LAUVERGNe J. J., Adalsteinsson S., 1976. Gènes pour la couleur de la toison de la brebis Corse. Ann. Génét. Sél. Anim., 8, 153-172.

Lauvergne J. J., Boyazoglu J. G., Carta R., Casu S., 1973. Caractéristiques démographiques de la race ovine Sarde. Ann. Génét. Sél. Anim., 5, 53-72.

LAUVERGNe J. J., Hoogschagen P., I978. Genetic formulas for the colours in the Texel, the Dutch and the Zwartbles sheep in the Netherlands. Ann. Génét. Sél. Anim., 10, 343-35r.

Lauvergne J. J., Rougeor J., Des Touches C., 1979. Coloured sheep and wool industries in France, in " Breeding coloured sheep and using coloured wool ". Proc. nat. Congr. Adelaide, South-Australia, South-Australian coloured sheep owners Society. Adelaide, I 27-I 29.

PRUD'HON M. H., I971. Étude de paramètres influençant la fécondité des brebis et la mortalité des agneaux d'un troupeau de race Mérinos d'Arles. Thèse, Université des Sciences et Techniques du Languedoc, Montpellier.

QUITTET E., 1965. Races ovines françaises. La Maison Rustique, Paris, $2^{\mathrm{e}}$ éd., retirage 1976, $64-69$.

ROUGEOT J., I980. Réflexions sur les productions de la laine et de la peau de mégisserie dans 1'élevage des moutons en France. Rapport à la Commission ovine du Plan. I.N.R.A., Laboratoire des Pelages et Fourrures, 78350 Jouy-en-Josas, 44 p. + annexes. Ronéoté.

SAcsos, 1979. Breeding coloured sheep and using coloured wool, National Congress Adelaide, South-Australia, South-Australian coloured sheep owners Association, Adelaide, S.A. Australia, 229 p.

WIENER G., I96r. Population dynamics in fourteen lowland breeds of Sheep in Great Britain. J. Agric. Sci., 57, 21-28. 